\title{
Elevated Cortisol Level and Cortisol/DHEAS Ratio in Schizophrenia as Revealed by Low-Dose Dexamethasone Suppression Test
}

\author{
Hiroaki Hori ${ }^{*}, 1,3$, Toshiya Teraishi ${ }^{1}$, Daimei Sasayama ${ }^{1}$, Takashi Fujii ${ }^{1}$, Kotaro Hattori ${ }^{1}$, \\ Masanori Ishikawa ${ }^{2}$ and Hiroshi Kunugi ${ }^{1,3}$ \\ ${ }^{I}$ Department of Mental Disorder Research, National Institute of Neuroscience, National Center of Neurology and \\ Psychiatry, 4-1-1, Ogawahigashi, Kodaira, Tokyo, 187-8502, Japan \\ ${ }^{2}$ National Center of Neurology and Psychiatry Hospital, Tokyo, 187-8502, Japan \\ ${ }^{3}$ CREST (Core Research of Evolutional Science \& Technology), JST (Japan Science and Technology Agency), Tokyo, \\ 102-0075, Japan
}

\begin{abstract}
Earlier studies have used the dexamethasone (DEX) suppression test (DST) to investigate the hypothalamicpituitary-adrenal (HPA) function in schizophrenia, although the findings are controversial. Recently there has been an increased interest in the role of dehydroepiandrosterone (DHEA) and its sulfate (DHEAS) in HPA axis function. Several studies have investigated basal DHEA(S) levels and cortisol/DHEA(S) ratios in schizophrenia patients, while no attempts have been made to investigate DHEA(S) level in response to the DEX administration. We aimed to compare the postDEX cortisol and DHEAS levels and the cortisol/DHEAS ratio between schizophrenia patients and healthy controls. Here we administered the DST to 43 patients with schizophrenia and 37 age- and sex-matched healthy controls. Plasma cortisol levels, serum DHEAS levels, and cortisol/DHEAS ratio after administration of $0.5 \mathrm{mg}$ of DEX were compared between the two groups. Schizophrenia patients showed significantly higher cortisol level and cortisol/DHEAS ratio than controls, while DHEAS levels were not significantly different between groups. These results suggest that besides the cortisol level, cortisol/DHEAS ratio as assessed with the DST might reflect abnormal HPA axis function in schizophrenia.
\end{abstract}

Keywords: Cortisol, dehydroepiandrosterone, dehydroepiandrosterone sulfate, dexamethasone suppression test, hypothalamicpituitary-adrenal axis, schizophrenia.

\section{INTRODUCTION}

The hypothalamic-pituitary-adrenal (HPA) axis is activated by all sorts of stressors, and this fact has represented the rationale for investigations into HPA axis function in schizophrenia, a disorder where stress could play a pivotal role in its onset and exacerbation. A large number of earlier studies have used the dexamethasone (DEX) suppression test (DST) to explore negative feedback inhibition of cortisol in schizophrenia patients. The findings were, however, controversial such that the rate of nonsuppression to the DST varied from $0 \%$ to $73 \%$ [1]. A metaanalysis of 26 DST studies [2] revealed that non-suppression rates of schizophrenia patients (19\%) were significantly lower than those of patients with major depression $(51 \%)$ and significantly higher than those of healthy controls (7\%). Indeed, HPA axis dysfunction is less consistently reported in schizophrenia than in depression $[3,4]$. On the other hand, studies employing psychosocial challenge paradigms have reported blunted cortisol responses in schizophrenia patients $[5,6]$. Individuals who are at risk for developing psychosis $[7,8]$, those with first episode psychosis $[9,10]$, and those

*Address correspondence to this author at the Department of Mental Disorder Research, National Institute of Neuroscience, National Center of Neurology and Psychiatry, 4-1-1, Ogawahigashi, Kodaira, Tokyo, 1878502, Japan; Tel: +81 42341 2711; Fax +81 42346 1744;

E-mail: hori@ncnp.go.jp with schizotypal personality $[11,12]$ have also been shown to be associated with altered HPA axis function, although findings are again not uniform, that is, both hyper- and hypocortisolism are reported. All these findings indicate that although schizophrenia would be associated with altered HPA axis function, its precise nature is yet to be established. More recently, there has been an increasing interest in the role of dehydroepiandrosterone (DHEA) and its more stable sulphated conjugated metabolite, DHEAS, in the neuroendocrinology of schizophrenia.

Like cortisol, DHEA and DHEAS (herein jointly referred to as "DHEA(S)"), are secreted by the adrenal cortex, at least partly in response to adrenocorticotropin-releasing hormone. DHEAS is the most abundant adrenal steroid in humans and its serum concentration is much higher than DHEA. DHEA(S) has neuroprotective actions, and is a precursor to more potent androgens and estrogens, such as testosterone and estradiol [13]. DHEA(S) is also classified as a neurosteroid, meaning that this hormone does not only cross the blood brain barrier to exert effects within central nervous system (i.e., a neuroactive steroid) but is synthesized de novo in the brain from its sterol precursor [14]. DHEA(S) acts as a gamma-aminobutyric acid type A receptor antagonist and is shown to be involved in the regulation of neuronal survival and differentiation [15]. It is also shown to act as a sigma-1 receptor agonist [16]. 
Importantly, DHEA(S) is known to possess antiglucocorticoid properties [17] because it antagonizes the effect of cortisol [13] and inhibits glucocorticoid-induced enzyme activity [18]. In healthy adults, acute administration of DHEA rapidly reduces cortisol levels [19]. Hence, DHEA(S) is assumed to be involved in stress responses [20] as well as in a broad range of behavioral functions [21]. Due to these characteristics, DHEA(S) has been implicated in the pathophysiology of various psychiatric disorders including major depression [22], bipolar disorder [23], dysthymic disorder [24], panic disorder [25], borderline personality disorder [26], eating disorder [27], posttraumatic stress disorder (PTSD) [28], and schizophrenia [29,30]. Several studies have investigated baseline DHEA(S) levels and cortisol/DHEA(S) ratios in schizophrenia patients, although their findings are not necessarily consistent. Nevertheless, based on the fact that the DHEA(S) level is influenced by the negative feedback of DEX administration, some studies have shown that DHEA(S) and/or cortisol/DHEA(S) ratio in response to the DST could be a sensitive marker for HPA axis function [27,31]. However, no studies to date have investigated DHEA(S) as assessed by the DST in schizophrenia.

In this context, the present study aimed to compare the post-DEX cortisol and DHEAS levels and the cortisol/DHEAS ratio between schizophrenia patients and age- and sex-matched healthy controls. We hypothesized that, among these measures, the cortisol/DHEAS ratio would be the most sensitive marker that distinguishes patients from healthy controls. We also expected that schizophrenia patients would be more likely to be associated with either or both of hyper- and hypo-cortisolism as compared to controls.

\section{METHODS}

\section{Participants}

Forty-three patients with schizophrenia (age range: 16-70 years), who were under treatment at the National Center of Neurology and Psychiatry Hospital or at a nearby hospital or psychiatric clinic, were enrolled. Of the 43 patients, 23 were hospitalized in the emergency ward of the National Center of Neurology and Psychiatry Hospital for the acute treatment of their psychotic symptoms at the time of the neuroendocrine testing. Consensus diagnoses for DSM-IV schizophrenia [32] were made by psychiatrists based on clinical interviews, observations and case notes. Thirty-seven age- and sexmatched healthy volunteers (age range: 23-70 years) were recruited from the community, through advertisements in free local magazines and our website announcement. At the first visit, the healthy participants were interviewed using the Japanese version of the Mini-International Neuropsychiatric Interview [33] by a research psychiatrist, and only those who demonstrated no current Axis I psychiatric disorders were enrolled in this study. In addition, those who demonstrated one or more of the following conditions during a nonstructured interview performed by an experienced psychiatrist were excluded from the healthy control group: past or current contact to psychiatric services, and other obvious self-reported signs of past primary psychotic and mood disorders as well as PTSD. Additional exclusion criteria from both the patient and control groups were: having a prior medical history of central nervous system disease or severe head injury, having a history of substance dependence or substance abuse within the past six months, having major systemic medical illnesses, or taking corticosteroids, antihypertensive medications, oral contraceptives or estrogen replacement therapies. The present experiments on our subjects were conducted in accordance with the Declaration of Helsinki. After the nature of the study procedures had been explained, written informed consent was obtained from all subjects. The study was approved by the ethics committee of the National Center of Neurology and Psychiatry.

\section{Dexamethasone Suppression Test}

First, participants took $0.5 \mathrm{mg}$ tablet of DEX orally at 23:00 h. For inpatients, a ward nurse gave this tablet to each patient. For the remaining subjects, compliance was monitored at the time of the blood collection by asking them whether they took the tablet as directed on the previous night. On the next day, plasma and serum samples were collected at 10:00 h. Plasma concentrations of cortisol were measured by radioimmunoassay and serum concentrations of DHEAS were measured by chemiluminescent enzyme immunoassay at SRL Corporation (Tokyo, Japan). The detection limit for cortisol was $27.59 \mathrm{nmol} / \mathrm{l}(=1.0 \mu \mathrm{g} / \mathrm{dl})$. Cortisol values under the detection limit were treated as 0 $\mathrm{nmol} / \mathrm{l}$. As our hypothesis was that the two extreme ends of cortisol values (i.e., both exaggerated and blunted cortisol reactivity) would be related to schizophrenia, in the main analysis we also adopted the categorical division of participants based on a priori defined cut-off values of cortisol, i.e., $27.59 \mathrm{nmol} / 1(=1.0 \mu \mathrm{g} / \mathrm{dl})$ and $137.95 \mathrm{nmol} / 1$ (= $5.0 \mu \mathrm{g} / \mathrm{dl}$ ), which were derived from several previous studies [12,34-36]. 'Non-suppressors' were defined to be individuals whose cortisol level was equal to or more than 137.95 nmol/l. 'Enhanced-suppressors' were defined as those individuals whose cortisol level was less than $27.59 \mathrm{nmol} / \mathrm{l}$ which corresponded to the cortisol level under the detection limit. The remaining individuals were considered to be 'moderate-suppressors'.

\section{Clinical Assessment, Antipsychotic Medication, and Psychological Distress}

For schizophrenia patients, symptoms were assessed by an experienced research psychiatrist in 41 of the total 43 patients using the Positive and Negative Syndrome Scale (PANSS) [37]; this yields a total score in addition to scores on positive, negative, and general psychopathology subscales. All patients with schizophrenia were receiving antipsychotics at the time of the neuroendocrine testing. Daily doses of antipsychotics, including depot antipsychotics, were converted to chlorpromazine equivalents using guidelines $[38,39]$.

For healthy controls, subjectively perceived psychological distress during one week preceding the neuroendocrine test was assessed via the Hopkins Symptom Checklist (HSCL) [40], a self-report questionnaire consisting of 58 (or 54) items which are scored on five underlying symptom dimensions, i.e., somatization, obsessivecompulsive, interpersonal sensitivity, anxiety, and depression symptoms. In the present study a validated Japanese version of the HSCL [41] comprising 54 items was 
used, as described in our previous study [35]. In this questionnaire, subjects were instructed to rate each item based on the distress perceived during the previous week, using a four-point likert scale, with "not-at-all" being scored 1, "occasionally", 2, "sometimes", 3, and "frequently", 4.

\section{Statistical Analysis}

Averages are reported as means \pm standard deviation (SD). To compare categorical variables, $\chi^{2}$ test was used. The t-test or Mann-Whitney U-test was used to examine differences between two groups. Plasma cortisol levels, serum DHEAS levels, and cortisol/DHEAS ratio were compared between two groups using the Mann-Whitney $U$ test because these hormonal data did not satisfy the assumptions for parametrical testing, which was revealed by the Kolmogorov-Smirnov test. Correlation between hormonal measures and clinical variables were calculated using the Spearman's rank correlation test. Statistical significance was set at two-tailed $\mathrm{p}<0.05$. Analyses were performed using the Statistical Package for the Social Sciences (SPSS) version 18.0 (SPSS Japan, Tokyo).

\section{RESULTS}

\section{Relationships of Hormonal Measures with Demographic Characteristics}

Table 1 shows the demographic and clinical characteristics of patients with schizophrenia and healthy controls. Patients and controls were well matched for age and sex. In the patient group, age significantly correlated with the DHEAS level $(\rho=-0.39, p=0.009)$, but not with cortisol level $(\rho=0.05, p=0.76)$ or cortisol/DHEAS ratio $(\rho$ $=0.26, \mathrm{p}=0.09)$. In the control group, age significantly correlated with the DHEAS level $(\rho=-0.55, p<0.001)$ and
cortisol/DHEAS ratio $(\rho=0.41, \mathrm{p}=0.013)$, but not with cortisol level $(\rho=0.12, p=0.49)$. Schizophrenic males and females did not significantly differ in cortisol level (MannWhitney $U=259.5, \mathrm{p}=0.44$ ) or cortisol/DHEAS ratio (Mann-Whitney $U=298.0, p=0.09$ ), whereas DHEAS was significantly higher in males than in females (Mann-Whitney $\mathrm{U}=120.0, \mathrm{p}=0.008$ ). Similarly, control males and females did not significantly differ in cortisol level (Mann-Whitney $\mathrm{U}=154.0, \mathrm{p}=0.62$ ) or cortisol/DHEAS ratio (MannWhitney $\mathrm{U}=181.0, \mathrm{p}=0.74)$, whereas DHEAS was significantly higher in males than in females (Mann-Whitney $\mathrm{U}=96.5, \mathrm{p}=0.025)$. Patients were significantly more likely to be smokers than controls; however, smokers and nonsmokers did not significantly differ in the cortisol level, DHEAS level, or cortisol/DHEAS ratio for both patients (all $\mathrm{p}>0.1$ ) and controls (all $\mathrm{p}>0.3$ ). Patients were significantly more likely to have a family history of psychiatric disorders than controls, although the presence $v s$ absence of such a history did not significantly impact on any of the three hormonal measures for both patients (all $p>0.4$ ) and controls (all $p>0.1$ ). In addition, all demographic and clinical variables were compared between males and females within each of the two diagnostic groups, and found no significant sex differences in any of the variables examined (all $\mathrm{p}>0.05)$.

\section{Relationships of Hormonal Measures with Clinical Variables and Symptoms}

Clinical variables including age at onset of schizophrenia, antipsychotic dosage, duration of antipsychotic medication, and duration of hospitalizations were not significantly correlated with any of the three hormonal measures, i.e., cortisol level, DHEAS level, and cortisol/DHEAS ratio (all $\mathrm{p}>0.05$ ). In total, 5 patients were

Table 1. Demographic Characteristics and Clinical Variables of Schizophrenia Patients and Control Subjects

\begin{tabular}{|c|c|c|c|c|}
\hline Sex, male/female ( $\%$ female) & $24 / 19(44 \%)$ & $20 / 17(46 \%)$ & $\chi 2(1)=0.02$ & 0.87 \\
\hline Smoking status, smokers/non-smokers & $18 / 25$ & $6 / 31$ & $\chi 2(1)=6.23$ & 0.013 \\
\hline Family history of psychiatric disorder, yes/no & $18 / 25$ & $5 / 32$ & $\chi 2(1)=7.80$ & 0.005 \\
\hline Duration of illness, years & $16.1 \pm 11.5$ & & & \\
\hline Duration of antipsychotic medication, years & $14.9 \pm 11.5$ & & & \\
\hline Chlorpromazine equivalents of antipsychotics, mg/day & $634.2 \pm 615.7$ & & & \\
\hline Number of hospitalizations & $3.6 \pm 3.3$ & & & \\
\hline PANSS positive score & $13.9 \pm 5.2$ & & & \\
\hline PANSS negative score & $17.1 \pm 7.8$ & & & \\
\hline PANSS general psychopathology score & $31.9 \pm 10.4$ & & & \\
\hline
\end{tabular}


taking typical antipsychotic(s), 29 were taking atypical antipsychotic(s) and 9 were taking both types at the time of testing. No significant differences were seen between these three medication groups in any of the three hormonal measures (all $p>0.6)$. Duration of illness was significantly negatively correlated with DHEAS level $(\rho=-0.33, p=$ 0.033 ), which was considered a reflection of a confounding effect of age because age was significantly correlated negatively with DHEAS level and positively with illness duration $(\mathrm{p}<0.001)$. Number of hospitalizations was significantly correlated negatively with DHEAS level $(\rho=-$ $0.36, p=0.020)$ and positively with cortisol/DHEAS ratio $(\rho$ $=0.33, \mathrm{p}=0.031)$. Outpatients and inpatients did not differ in any of the three hormonal measures (all $\mathrm{p}>0.2$ ). Symptom dimensions as assessed with the PANSS, i.e., positive symptoms, negative symptoms, general psychopathology and total score of PANSS, were not significantly correlated with any of the hormonal measures (all $\mathrm{p}>0.2$ ). In healthy controls, no significant associations were seen between the three hormonal outcomes and any of the five symptom dimensions of the HSCL (all $\mathrm{p}>0.1$ ).

\section{Comparisons of Hormonal Measures between Patients vs Controls}

Results of cortisol and DHEAS levels and cortisol/DHEAS ratio (multiplied by 100) for schizophrenia patients and healthy controls are provided in Fig. (1). Patients showed significantly higher cortisol level and cortisol/DHEAS ratio, while no significant difference was seen in the DHEAS level. As for the suppression pattern, patients showed a greater ratio of non-suppression of cortisol than controls at a non-significant trend level $\left(\chi^{2}(1)=3.35, p\right.$ $=0.067)$ while no significant differences were seen between the two groups in the ratio of enhanced-suppression $\left(\chi^{2}(1)=\right.$ $1.55, \mathrm{p}=0.21)$. Compared to controls, outpatients showed significantly higher cortisol level (Mann-Whitney $\mathrm{U}=503.0$, $\mathrm{p}=0.025$ ) but not cortisol/DHEAS ratio (Mann-Whitney $\mathrm{U}$ $=460.0, \mathrm{p}=0.13)$ while inpatients showed significantly higher cortisol level (Mann-Whitney $U=598.0, p=0.009$ ) and cortisol/DHEAS ratio (Mann-Whitney $\mathrm{U}=563.5, \mathrm{p}=$ 0.036).

Table 2 shows a comparison of findings from studies that examined both cortisol and DHEA and/or DHEAS levels in schizophrenia/first-episode psychosis. Of these nine studies, three [42-44] did not provide cortisol/DHEA(S) ratio. It is clear from this table that previous findings of these hormonal indices in schizophrenia have been variable, such that some studies reported an elevation of these hormonal indices in schizophrenia while others not.

\section{DISCUSSION}

The present study found that the cortisol level and cortisol/DHEAS ratio, in response to the $0.5 \mathrm{mg}$ DEX administration, were significantly higher in schizophrenia patients as compared to healthy controls. The DHEAS level was not significantly different between the two groups. These hormonal indices were not significantly associated with the antipsychotic dosage or symptom dimensions, while cortisol/DHEAS ratio was to some extent associated with the number of hospitalizations and outpatient/inpatient status. (a)

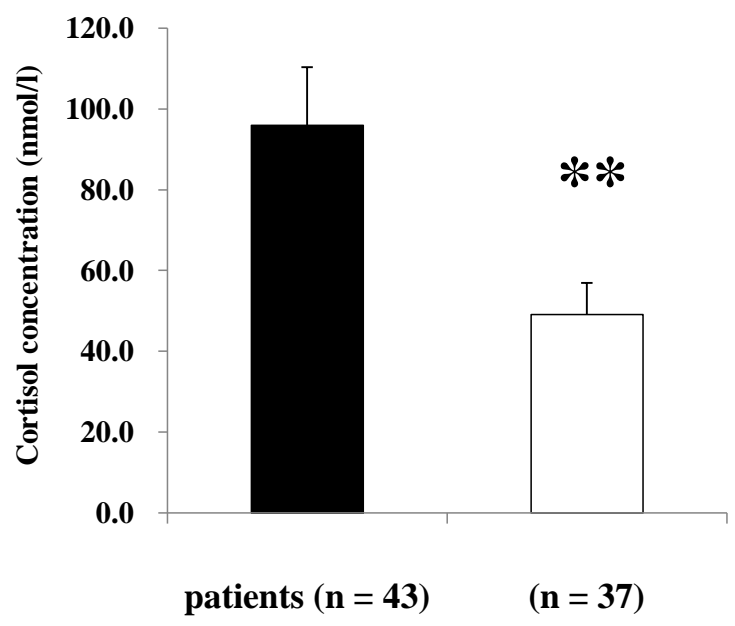

(b)

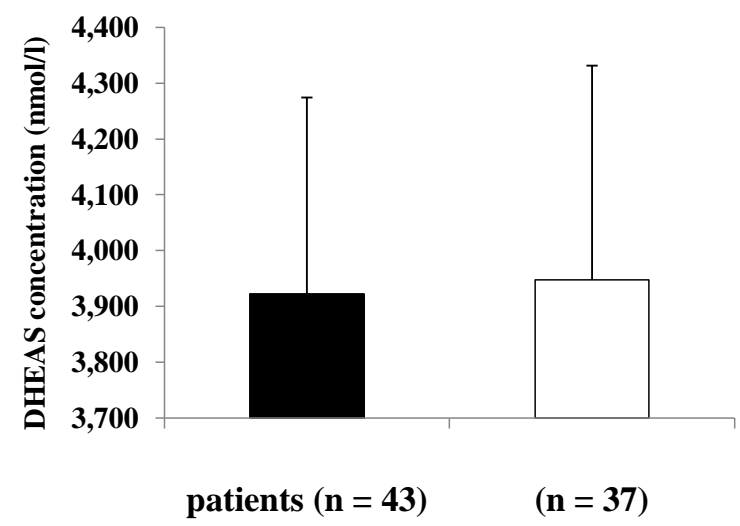

(c)

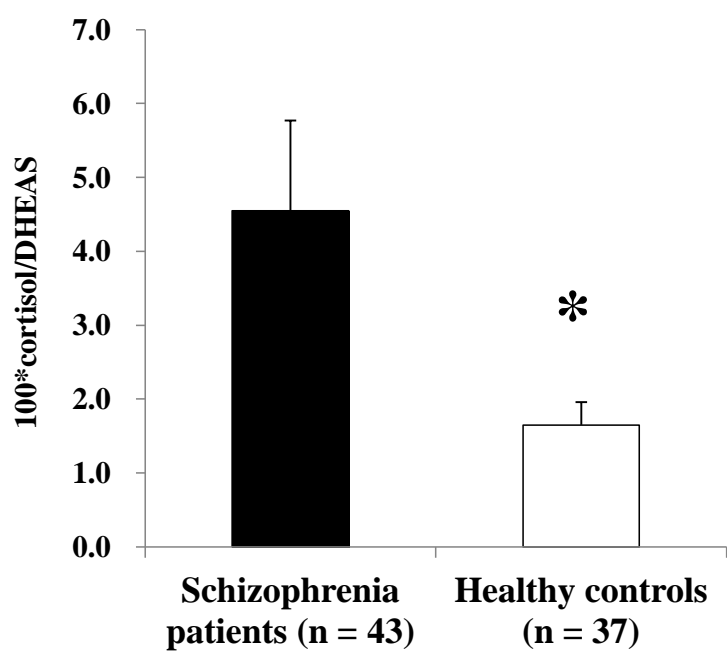

Fig. (1). Hormonal data, including cortisol level (a), DHEAS level (b) and $100 *$ cortisol/DHEAS ratio (c), of schizophrenia patients (black bars) and healthy control subjects (white bars). $* \mathrm{p}<0.05$; $* * \mathrm{p}<0.01$ (by Mann-Whitney U test). Error bars represent standard errors of the mean. 
Table 2. Summary of Studies that Investigated Both Cortisol and DHEA(S) Levels in schizophrenia Patients and Healthy Controls

\begin{tabular}{|c|c|c|c|c|c|c|c|c|}
\hline & \multicolumn{2}{|c|}{ Number of Sample } & Matching Status & Patient Characteristics & Sampling Method & \multicolumn{3}{|c|}{ Hormonal Outcomes (Patients vs Controls) } \\
\hline Shirayama et al. (2002) & 14 & 13 & males only ${ }^{c}$ & moderate negative symptoms & basal & $\uparrow \uparrow^{e}$ & N.S. & N.A. \\
\hline Ritsner et al. (2004) & 40 & 15 & age/sex & chronically ill, hospitalized & basal & N.S. & N.S. & $\uparrow \uparrow$ \\
\hline Strous et al. (2004) & 37 & 25 & age/sex & first-episode & basal & N.S. & $\uparrow \uparrow$ & N.S. \\
\hline Ritsner et al. $(2007)^{\mathrm{a}}$ & 43 & 20 & age/sex & chronically ill, hospitalized & basal & $\uparrow \uparrow$ & $\uparrow^{\mathrm{f}}$ & $\uparrow \uparrow$ \\
\hline Yilmaz et al. (2007) & 66 & 28 & age/sex(males only) & chronic & basal & $\uparrow \uparrow$ & N.S. & N.A. \\
\hline Gallagher et al. (2007) & 20 & 20 & age/sex & probably chronic & basal $^{\mathrm{d}}$ & $\uparrow \uparrow$ & $\uparrow \uparrow$ & N.S. \\
\hline
\end{tabular}

$\uparrow \uparrow:$ significantly higher in schizophrenia patients

N.A.: not applicable

N.S.: not significant

${ }^{a}$ Samples were collected at baseline, after 2 , and 4 weeks.

${ }^{\mathrm{b}}$ Hormones were measured at baseline and after 12 weeks (baseline/after 12 weeks).

${ }^{\mathrm{c}}$ Age not matched.

${ }^{\mathrm{d}}$ Serial sampling from 13:00 h to $16: 00 \mathrm{~h}$.

${ }^{\mathrm{e}}$ Not significant between schizophrenia patients with low negative symptoms and controls.

fDHEAS level was significantly higher in schizophrenia patients, while no significant difference was observed in DHEA level.

As described earlier, impaired negative feedback of HPA axis has been implicated in the pathophysiology of schizophrenia, yet findings on cortisol levels from DST studies were not consistent. As shown in Table 2, studies that investigate both cortisol and DHEA(S) basal levels in schizophrenia patients have again yielded mixed findings, not only for cortisol and DHEA(S) levels but also for cortisol/DHEA(S) ratio. This controversy might be attributable, at least in part, to a number of differential demographic and/or clinical characteristics across studies, such as age, symptom severity, medication status, and comorbid psychiatric disorders, given that these variables are shown to affect cortisol and DHEA(S) levels. For instance, Ritsner et al. [45] first investigated cortisol/DHEA(S) ratio in schizophrenia patients in comparison to healthy controls and found this ratio to be significantly elevated in the patient group. This research group confirmed the elevated cortisol/DHEA(S) ratio in their subsequent study where hormonal levels were measured three times every two weeks [46]. In contrast, Gallagher et al. [30] did not find such a significant difference in the cortisol/DHEA ratio between schizophrenia patients and healthy controls. In addition, two studies that examined this ratio in individuals with firstepisode psychosis did not observe significant differences in this ratio between these individuals and controls $[9,47]$.

Against this background, we administered the DST to measure DHEAS as well as cortisol levels in chronic schizophrenia patients. The significantly elevated cortisol levels in patients than in controls, together with similar levels of DHEAS between the two groups, resulted in the significantly higher cortisol/DHEAS ratio in patients. Similar to the robust effect of oral DEX administration on cortisol suppression, the suppressive effect of DEX on DHEA(S) has been demonstrated [27,31]. Taken together, whereas our result of the elevated cortisol level in schizophrenia patients indicates overall impaired negative feedback inhibition of HPA axis in schizophrenia, the similar level of DHEAS in the two diagnostic groups might suggest that circulating DHEAS level is regulated by several other mechanisms as well as the negative feedback inhibition. However, the mechanism of this dissociation between cortisol and DHEA(S) levels is unclear. Nevertheless, our findings imply that HPA axis hyperactivity as indexed by the elevated cortisol level in schizophrenia is not compensated by the putative anti-glucocorticoid effect of DHEAS, thereby possibly leading to the persistent stress vulnerability in patients with chronic schizophrenia. It may also be worth noting that, among the five studies that have investigated cortisol/DHEAS ratio in patients with schizophrenia/psychosis, three studies including ours that examined chronically ill schizophrenia patients showed significantly higher cortisol/DHEAS ratio in patients than in controls $[45,46]$ while two studies examining individuals with first-episode psychosis found no significant differences $[9,47]$. The cortisol/DHEAS ratio might thus vary depending on disease stages of schizophrenia, with this ratio becoming higher as the stage progresses.

With respect to the relationship between clinical variables and hormonal levels in schizophrenia patients, we found that, among a number of clinical variables, only those associated with hospital admission (i.e., the number of hospitalizations and the present status of in-/out-patient) were significantly related to altered HPA axis function; hospitalization was associated with higher cortisol/DHEAS ratio. It may be that although an alteration in HPA axis function is present independent of clinical states of schizophrenia patients, which is in line with a previous finding [46], acutely ill inpatients would exhibit even greater alteration. To draw any conclusion, further studies, longitudinal follow-up studies in particular, are required.

Another important issue that should be taken into consideration is the protocol for DST, i.e., the dose of DEX used for the pretreatment as well as the time of hormonal 
assays after DEX administration. As for the dose of DEX, most of the earlier studies in schizophrenia have used $1.0 \mathrm{mg}$ of DEX to assess negative feedback of HPA axis [1]. However, we herein administered a lower dose of DEX (i.e., $0.5 \mathrm{mg}$ ) to make the sensitivity high for both incomplete and enhanced suppression of cortisol, taking into account a growing body of evidence indicating that HPA axis abnormalities consist of hyper- and hypo-cortisolism. This approach, that is the DST using a low dose DEX, has successfully been employed in the studies of patients with PTSD to detect their enhanced negative feedback of cortisol $[48,49]$. Although we did not find any excess of enhanced suppression in schizophrenia patients as compared to controls, the fact that we were able to detect the altered HPA axis function in schizophrenia would point to the usefulness of DST with a low dose DEX for this population as well. Regarding the time of hormonal measurements, we decided to draw blood samples at 10:00 $\mathrm{h}$ because previous DST studies that have looked at enhanced suppression of cortisol using low-dose DEX draw blood in the morning $[48,49]$. However, such differences in the dose of DEX and the time of hormonal measurements should be taken into account when comparing the present results with previous ones.

Several limitations need to be commented upon. First, as we did not include baseline measurements of cortisol and DHEAS levels, the extent to which each participant suppressed his/her cortisol and DHEAS in response to the $0.5 \mathrm{mg}$ of DEX cannot be determined. Second, as we sampled blood for hormone measurements only at a single point, the diurnal variation of the hormone levels was unknown. Third, since all patients were receiving antipsychotics, such medication may have influenced HPA axis function as has been demonstrated [50,51]. Although we did not find any significant correlations between antipsychotic dosage and hormonal measures, we have to acknowledge that our data on the chlorpromazine equivalents are themselves limited in that the inpatients were in the midst of their acute treatment and thus the prescription of antipsychotics tended to be frequently changed around the time of the hormone measurement. It should also be noted that chlorpromazine equivalents are an approximate indicator of D2 receptor antagonist activity and most antipsychotics have multiple effects that could have variable influence on the HPA axis function. Fourth, HPA axis abnormalities have been reported in a variety of psychiatric disorders, particularly in mood disorders, it is not known whether the altered cortisol level and cortisol/DHEAS ratio observed here represent schizophrenia-specific HPA axis dysfunction or rather common HPA axis alteration in relation to stressful conditions in general. Finally, we did not collect data on the menstrual cycle or history of childhood trauma, both of which are shown to moderate HPA axis function.

To sum, the present study found that HPA axis function in schizophrenia is altered, as indicated by the elevated cortisol level and cortisol/DHEAS ratio in response to the low-dose DEX administration. In addition to the cortisol level, cortisol/DHEAS ratio may reflect some aspect of HPA axis abnormalities in schizophrenia. Future studies that examine these hormones both before and after the DEX administration are needed to disentangle the baseline and feedback components of the HPA axis alteration in schizophrenia.

\section{ACKNOWLEDGEMENTS}

H.H. was supported by Grant-in-Aid for Young Scientists from the Japan Society for the Promotion of Science. H.K. was supported by Core Research for Evolutional Science and Technology (CREST), Japan Science and Technology Agency (JST), Health and Labor Sciences Research Grants (Comprehensive Research on Disability, Health, and Welfare), and Intramural Research Grant for Neurological and Psychiatric Disorders of National Center of Neurology and Psychiatry.

\section{CONFLICT OF INTEREST}

None declared.

\section{REFERENCES}

[1] Tandon R, Mazzara C, DeQuardo J, et al. Dexamethasone suppression test in schizophrenia: Relationship to symptomatology, ventricular enlargement, and outcome. Biol Psychiatry 1991;29: 953-64.

[2] Sharma RP, Pandey GN, Janicak PG, et al. The effect of diagnosis and age on the DST: a metaanalytic approach. Biol Psychiatry 1988; 24: 555-68.

[3] Cotter D, Pariante CM. Stress and the progression of the developmental hypothesis of schizophrenia. Br J Psychiatry 2002; 181: 363-5.

[4] Holsboer F. In: Grossman A, Ed. Clinical endocrinology. Oxford: Blackwell Science 1998; pp.1096-116.

[5] Brenner K, Liu A, Laplante DP, et al. Cortisol response to a psychosocial stressor in schizophrenia: blunted, delayed, or normal? Psychoneuroendocrinology 2009; 34: 859-68.

[6] Jansen LM, Gispen-de Wied CC, Kahn RS. Selective impairments in the stress response in schizophrenic patients. Psychopharmacology (Berl) 2000; 149: 319-25.

[7] Thompson KN, Berger G, Phillips LJ, et al. HPA axis functioning associated with transition to psychosis: combined DEX/CRH test. J Psychiatr Res 2007; 41: 446-50.

[8] Walker EF, Brennan PA, Esterberg M, et al. Longitudinal changes in cortisol secretion and conversion to psychosis in at-risk youth. J Abnorm Psychol 2010; 119: 401-8.

[9] Garner B, Phassouliotis C, Phillips LJ, et al. Cortisol and dehydroepiandrosterone-sulphate levels correlate with symptom severity in first-episode psychosis. J Psychiatr Res 2011; 45: 24955 .

[10] Ryan MC, Sharifi N, Condren R, Thakore JH. Evidence of basal pituitary-adrenal overactivity in first episode, drug naive patients with schizophrenia. Psychoneuroendocrinology 2004; 29: 1065-70.

[11] Mitropoulou V, Goodman M, Sevy S, et al. Effects of acute metabolic stress on the dopaminergic and pituitary-adrenal axis activity in patients with schizotypal personality disorder. Schizophr Res 2004; 70: 27-31.

[12] Hori H, Teraishi T, Ozeki Y, et al. Schizotypal personality in healthy adults is related to blunted cortisol responses to the combined dexamethasone/ corticotropin-releasing hormone test. Neuropsychobiology 2011; 63: 232-41.

[13] Maninger N, Wolkowitz OM, Reus VI, Epel ES, Mellon SH Neurobiological and neuropsychiatric effects of dehydroepiandrosterone (DHEA) and DHEA sulfate (DHEAS). Front Neuroendocrinol 2009; 30: 65-91.

[14] Baulieu EE, Robel P. Dehydroepiandrosterone (DHEA) and dehydroepiandrosterone sulfate (DHEAS) as neuroactive neurosteroids. Proc Natl Acad Sci USA 1998; 95: 4089-91.

[15] Regelson W, Kalimi M. Dehydroepiandrosterone (DHEA)--the multifunctional steroid. II. Effects on the CNS, cell proliferation, metabolic and vascular, clinical and other effects: mechanism of action? Ann N Y Acad Sci 1994; 719: 564-75.

[16] Maurice T, Gregoire C, Espallergues J. Neuro(active)steroids actions at the neuromodulatory sigmal (sigma1) receptor: Biochemical and physiological evidences, consequences in neuroprotection. Pharmacol Biochem Behav 2006; 84: 581-97.

[17] Kalimi M, Shafagoj Y, Loria R, Padgett D, Regelson W. Antiglucocorticoid effects of dehydroepiandrosterone (DHEA). Mol Cell Biochem 1994; 131: 99-104. 
[18] Browne ES, Wright BE, Porter JR, Svec F. Dehydroepiandrosterone: antiglucocorticoid action in mice. Am J Med Sci 1992; 303: 366-71.

[19] Wolf OT, Koster B, Kirschbaum C, et al. A single administration of dehydroepiandrosterone does not enhance memory performance in young healthy adults, but immediately reduces cortisol levels. Biol Psychiatry 1997; 42: 845-8.

[20] Charney DS. Psychobiological mechanisms of resilience and vulnerability: implications for successful adaptation to extreme stress. Am J Psychiatry 2004; 161: 195-216.

[21] Rupprecht R, Holsboer F. Neuroactive steroids: mechanisms of action and neuropsychopharmacological perspectives. Trends Neurosci 1999; 22: 410-6.

[22] van Broekhoven F, Verkes RJ. Neurosteroids in depression: a review. Psychopharmacology (Berl) 2003; 165: 97-110.

[23] Marx CE, Stevens RD, Shampine LJ, et al. Neuroactive steroids are altered in schizophrenia and bipolar disorder: relevance to pathophysiology and therapeutics. Neuropsychopharmacology 2006; 31: 1249-63.

[24] Markianos M, Tripodianakis J, Sarantidis D, Hatzimanolis J. Plasma testosterone and dehydroepiandrosterone sulfate in male and female patients with dysthymic disorder. J Affect Disord 2007; 101: 255-8.

[25] Brambilla F, Mellado C, Alciati A, et al. Plasma concentrations of anxiolytic neuroactive steroids in men with panic disorder. Psychiatry Res 2005; 135: 185-90.

[26] Jogems-Kosterman BJ, de Knijff DW, Kusters R, van Hoof JJ. Basal cortisol and DHEA levels in women with borderline personality disorder. J Psychiatr Res 2007; 41: 1019-26.

[27] Monteleone P, Luisi M, Martiadis V, et al. Impaired reduction of enhanced levels of dehydroepiandrosterone by oral dexamethasone in anorexia nervosa. Psychoneuroendocrinology 2006; 31: 537-42.

[28] Yehuda R, Brand SR, Golier JA, Yang RK. Clinical correlates of DHEA associated with post-traumatic stress disorder. Acta Psychiatr Scand 2006; 114: 187-93.

[29] Ritsner M, Gibel A, Maayan R, et al. Cortisol/dehydroepiandrosterone ratio and responses to antipsychotic treatment in schizophrenia. Neuropsychopharmacology 2005; 30: 1913-22.

[30] Gallagher P, Watson S, Smith MS, Young AH, Ferrier IN. Plasma cortisol-dehydroepiandrosterone (DHEA) ratios in schizophrenia and bipolar disorder. Schizophr Res 2007; 90: 258-65.

[31] Maninger N, Capitanio JP, Mason WA, Ruys JD, Mendoza SP. Acute and chronic stress increase DHEAS concentrations in rhesus monkeys. Psychoneuroendocrinology 2010; 35: 1055-62.

[32] American Psychiatric Association. DSM-IV: diagnostic and statistical manual of mental disorders. $4^{\text {th }}$ ed. Washington DC: American Psychiatric Association 1994.

[33] Sheehan DV, Lecrubier Y, Sheehan KH, et al. The miniinternational neuropsychiatric interview (M.I.N.I.): the development and validation of a structured diagnostic psychiatric interview for DSM-IV and ICD-10. J Clin Psychiatry 1998; 59 (Suppl 20): S22-33.

[34] Kunugi H, Ida I, Owashi T, et al. Assessment of the dexamethasone/ $\mathrm{CRH}$ test as a state-dependent marker for hypothalamic-pituitaryadrenal (HPA) axis abnormalities in major depressive episode: a Multicenter Study. Neuropsychopharmacology 2006; 31: 212-20.
[35] Hori H, Ozeki Y, Teraishi $\mathrm{T}$, et al. Relationships between psychological distress, coping styles, and HPA axis reactivity in healthy adults. J Psychiatr Res 2010; 44: 865-73.

[36] Hori H, Teraishi T, Sasayama D, et al. Poor sleep is associated with exaggerated cortisol response to the combined dexamethasone/ CRH test in a non-clinical population. J Psychiatr Res 2011; 45: 1257-63.

[37] Kay SR, Fiszbein A, Opler LA. The positive and negative syndrome scale (PANSS) for schizophrenia. Schizophr Bull 1987; 13: 261-76.

[38] American Psychiatric Association. Practice Guidelines for the Treatment of Patients with Schizophrenia. Washington DC: American Psychiatric Press 1997.

[39] Inagaki A, Inada T, Fujii Y, Yagi G. Equivalent dose of psychotropics. Tokyo: Seiwa Shoten 1999 (in Japanese).

[40] Derogatis LR, Lipman RS, Rickels K, Uhlenhuth EH, Covi L. The Hopkins symptom checklist (HSCL): a self-report symptom inventory. Behav Sci 1974; 19: 1-15.

[41] Nakano K. Stress management. Tokyo: Kongo-syuppan 2005 (in Japanese).

[42] Shirayama Y, Hashimoto K, Suzuki Y, Higuchi T. Correlation of plasma neurosteroid levels to the severity of negative symptoms in male patients with schizophrenia. Schizophr Res 2002; 58: 69-74.

[43] Goyal RO, Sagar R, Ammini AC, Khurana ML, Alias AG. Negative correlation between negative symptoms of schizophrenia and testosterone levels. Ann N Y Acad Sci 2004; 1032: 291-4.

[44] Yilmaz N, Herken H, Cicek HK, Celik A, Yurekli M, Akyol O. Increased levels of nitric oxide, cortisol and adrenomedullin in patients with chronic schizophrenia. Med Princ Pract 2007; 16: $137-41$.

[45] Ritsner M, Maayan R, Gibel A, Strous RD, Modai I, Weizman A. Elevation of the cortisol/dehydroepiandrosterone ratio in schizophrenia patients. Eur Neuropsychopharmacol 2004; 14: 267-73.

[46] Ritsner M, Gibel A, Maayan R, et al. State and trait related predictors of serum cortisol to DHEA(S) molar ratios and hormone concentrations in schizophrenia patients. Eur Neuropsychopharmacol 2007; 17: 257-64.

[47] Strous RD, Maayan R, Lapidus R, et al. Increased circulatory dehydroepiandrosterone and dehydroepiandrosterone-sulphate in first-episode schizophrenia: Relationship to gender, aggression and symptomatology. Schizophr Res 2004; 71: 427-34.

[48] Grossman R, Yehuda R, New A, et al. Dexamethasone suppression test findings in subjects with personality disorders: Associations with posttraumatic stress disorder and major depression. Am J Psychiatry 2003; 160: 1291-8.

[49] Yehuda R, Golier JA, Halligan SL, Meaney M, Bierer LM. The ACTH response to dexamethasone in PTSD. Am J Psychiatry 2004; 161: 1397-403.

[50] Lammers CH, Garcia-Borreguero D, Schmider J, et al. Combined dexamethasone/corticotropin-releasing hormone test in patients with schizophrenia and in normal controls: II. Biol Psychiatry 1995 ; 38: 803-7.

[51] Venkatasubramanian G, Chittiprol S, Neelakantachar N, Shetty T, Gangadhar BN. Effect of antipsychotic treatment on insulin-like growth factor-1 and cortisol in schizophrenia: a longitudinal study. Schizophr Res 2010; 119: 131-7. 